\title{
Abstract: Leveraging Web Data for Skin Lesion Classification
}

\author{
Fernando Navarro ${ }^{1}$, Sailesh Conjeti ${ }^{2}$, Federico Tombari ${ }^{1}$, Nassir Navab $^{1,3}$ \\ ${ }^{1}$ Computer Aided Medical Procedures, Technische Universität München, Germany \\ 2 Deutsches Zentrum für Neurodegenerative Erkrankungen (DZNE), Bonn, Germany \\ ${ }^{3}$ Computer Aided Medical Procedures, Johns Hopkins University, USA \\ fernando.navarro@tum.de
}

The success of deep learning is mainly based on the assumption that for the given application, there is access to a large amount of annotated data. In medical imaging applications, having access to a big-well-annotated data-set is restrictive, time-consuming and costly to obtain. Although diverse techniques as data augmentation can be leveraged to increase the size and variability within the data-set, the representativeness of the training set is still limited by the number of available samples. Furthermore, a small-size and well-annotated data-set can not guarantee the generalizability to unseen samples.

As a consequence for the aforementioned problem, we have proposed in [1] to utilize the vast amount of free available data from the web to alleviate the need of a large-well-annotated data-set in the so-called Webly Supervised Learning methodology presented in [2]. Harvesting images from the web presents the opportunity to increase the variability and heterogeneity of the training set at the cost of label noise. These label noises include cross-domain: retrieved images opposite to the dermatology domain and cross-category: retrieved images visually similar to the query image yet belonging to a different class. To overcome the first type of noise we have proposed a search by image technique to increase the search specificity and retrieve only images visually similar to the query. The second type of noise is reduced by modeling the noise in the data-set with a classtransition matrix, estimated from the web-retrieved images as proposed in [3]. To the best of our knowledge, our work has been the first applying webly supervised learning in medical imaging. To validate our methodology, we have tested our system in the context of ten-class fine-grained skin lesion classification. Our results show that the proposed methodology increase the overall classification accuracy from $71.25 \%$ to $80.53 \%$ due to the web-supervision.

\section{References}

1. Navarro F, Conjeti S, Tombari F, et al. Webly supervised learning for skin lesion classification. Proc MICCAI. 2018; p. 398-406.

2. Chen X, Gupta A. Webly supervised learning of convolutional networks. Proc CVPR. 2015; p. 1431-1439.

3. Patrini G, Rozza A, Menon AK, et al. Making deep neural networks robust to label noise: a loss correction approach. Proc CVPR. 2017; p. 2233-2241. 